\title{
VOISINAGES: Démocratisation et géopolitique
}

Jacques Rupnik

"Nous devons faire l'Europe non seulement dans l'intérêt des pays libres, mais aussi pour pouvoir y accueillir les peuples de l'Est qui, délivrés des sujétions qu'ils ont subies jusqu' à présent, nous demanderont leur adhésion et notre appui moral". Robert Schuman (1956)'

L'Europe est aujourd'hui confrontée à une double crise: la crise de confiance interne dans son projet et la crise externe marquée par la déstabilisation simultanée des ses voisinages à l'Est comme au Sud. Répondre à la seconde dépendra de la capacité à surmonter la première. Dix ans après son grand l'élargissement à l'Est, l'Union européenne se trouve dans une situation paradoxale: une demande d'Europe dans sa périphérie orientale, de Kiev à Belgrade, au moment où le doute s'installe au centre même de l'Union. Les élections européennes du 25 mai 2014, marquées par le désenchantement voire la défiance envers l'Union européenne, contrastaient le même jour avec l'élection présidentielle à Kiev présentée et vécue comme un choix européen. L'Europe est décriée comme l'une des causes de la crise économique et identitaire à l'Ouest du continent, et perçue comme une réponse possible à la crise politique, économique et identitaire à l'Est. La crise de la zone euro a fait planer

Discours prononcé par Robert Schuman, ministre des affaires étrangères, à Luxembourg le 3 novembre 1956, en pleine révolution hongroise, mais cité dans France Forum seulement dans le numéro 52 de novembre 1963 sans préciser la date ni le contexte du propos. 
le spectre de la désintégration et favorisée le repli² au moment même où les bouleversements politiques en Ukraine, comme la recomposition post-yougoslave dans Balkans, font de l'Europe un point d'ancrage indispensable. II n'est pas aisé de se projeter vers la périphérie alors que le centre même est en doute.

Avec son élargissement à l'Est, l'Union européenne s'est découvert un nouveau voisinage oriental qui se trouve être aussi celui de la Russie qui le considère comme son "étranger proche" et sa sphère d'influence. A travers la crise déclenchée en novembre 2013 à l'occasion du sommet de Vilnius proposant un Accord d'Association à six pays postsoviétiques concernés par le Partenariat oriental, il apparaît clairement que l'UE n'avait pas anticipé à quel point l'attrait de son modèle était désormais considéré comme une menace par la Russie. Celle-ci n'a pas hésité au lendemain de la chute du régime lanoukovitch à Kiev à employer la force pour annexer la Crimée en mars 2014 et le levier économique pour imposer aux pays concernés un choix qu'ils auraient préféré éviter entre l'Accord d'Association avec I'UE signé à Bruxelles le 27 juin par l'Ukraine, la Géorgie, la Moldavie et le projet russe d'une Union Economique Eurasienne lancé le 29 mai 2014 par Poutine avec les présidents du Belarus et du Kazakhstan./NOTE/ Deux projets d'intégration régionale concurrents sont clairement en présence, voisinage européen contre espace post-soviétique, où les enjeux de démocratisation et de géopolitique se trouvent étroitement imbriqués. L'Europe doit désormais repenser ce double enjeu: l'orientation européenne peut être à la périphérie de l'Union un vecteur de démocratisation, mais lorsque celleci amène au pouvoir des gouvernements réformateurs et tournés vers l'Ouest le voisinage peut aussi devenir un espace la rivalité et même de conflit avec la Russie.

2 A côté de l'abondante littérature eurosceptique la nouveauté est que la perspective de la désintégration de l'UE est abordée explicitement par des universitaires et des experts favorables au projet européen tels que François Heisbourg, La fin du rêve européen, Paris, Stock, 2013; Jacques Lesourne, L'Europe à l'heure de son crépuscule, Paris, O. Jacob, 2013; Jan Zielonka, professeur d'études européennes à l'Université d'Oxford, Is Europe Doomed? Oxford, Polity Press, 2014. L'Institut des Sciences Humaines (Institut für die Wissenschaften von den Menschen) à Vienne organisa en 2012 et 2013 plusieurs colloques comparant le processus de désintégration de l'UE à ceux qu'avaient connu I'Union soviétique ou l'Empire des Habsbourg, cf. Ivan Krastev, "European dis-Union: lessons from the Soviet collapse", www. opendemocracy.net 15.mai 2012et Learning from the Habsburg Experience, IWMpost,(Vienne), n 111 (sept. 2012-avril 2013). L'historien David Engels fait le parallèle entre la crise de l'Europe et la chute de Rome antique dans Le Declin. La crise de I'Union europénne et la chute de la République romaine, Paris, éd. du Toucan, 2014. Cf. aussi le numéro spécial de la revue Aspen Europe, Breakup of Europe (n 1, 2014). Sur le déclin européen et la perte d'influence de l'UE au plan international cf. Richard Youngs, Europe's Decline and Fall: the struggle against global irrelevance, Profile Books, 2010; Z.Laidi, Le reflux de l'Europe, Paris, Presses de Sciences Po, 2013. Cette littérature europessimiste offre un contraste saisissant avec l' "europhorie" qui prévalait une décennie auparavant au moment de l'élargissement de l'UE avec, notamment, Jeremy Rifkin, The European Dream, how Europe's vision of the future is quietly eclipsing the American dream, London, Penguin, 2004 ou Mark Leonard, Why Europe will run the XXIst century, London, Public Affairs, 2005. 
Une dimension géopolitique inavovée de la démocratisation était en fait inhérente à l'élargissement à l'Est de l'UE en 2004. Etendre à l'Est du continent la "zone de prospérité, de démocratie et de paix", selon la formule consacrée, posait d'emblée la question de la relation future avec le voisinage postsoviétique et donc avec la Russie. L'UE peut-elle donner une réponse partagée à cette question sans que celle-ci ne se résume à la recherche du plus petit dénominateur commun? Cela pose aussi à l'Union la question des ses frontières: jusqu'où peut-elle, doit-elle s'élargir? Quel type de frontières à la périphérie de I'UE? En passant, par élargissements successifs, de 6 à 28 membres, I'UE devenait plus représentative du continent européen. Fallait-il à terme viser la Grande Europe et envisager de faire coïncider les frontières de l'UE avec celles de l'Europe? Elles sont clairement définies au Nord, au Sud et à l'Ouest, mais floues et contestées à l'Est. Ouverture maximum à l'Est, Russie comprise, ce fut l'option du Conseil de l'Europe dès le lendemain de la guerre froide. Le projet de Confédération européenne proposé par François Mitterrand en 1990 aux pays d'Europe Centrale voulait à la fois repousser à plus tard la perspective de leur adhésion à l'UE et ménager la relation avec la Russie soviétique de Gorbatchev ${ }^{3}$. Le projet échova précisément pour cette raison: les pays d'Europe centrale sortant du Pacte de Varsovie n'avaient aucune envie de se retrouver dans un même ensemble politique avec une Union soviétique en train d'éclater. Eł la France ne retrouva plus, dans la recherche d'une nouvelle architecture européenne, un second souffle depuis cette tentative à la fois visionnaire et inadaptée.

Elargir jusqu'où? Une réponse symboliquement forte pourrait être "de la Baltique à l'Adriatique" pour reprendre la formule de Churchill dans son discours célèbre de 1946 sur le "rideau de fer" qui divisait l'Europe; plus plausible et rassurante sans doute que la vision américaine proposée par Victoria Nulen, la sous-secrétaire d'Ełat aux affaires européennes des Ełats-Unis : "De Dayton à Donetsk, l'Europe unie et libre" ${ }^{4} .$.

\section{Centre et périphéries}

Une autre façon d'appréhender le décalage entre l'influence de I'UE

3 Jacques Rupnik, "La France de François Mitterrand face aux changements en Europe de l'Est" in S. Cohen (dir.) La politique étrangère de François Mitterrand face à 1989, Paris, Sevil, 1998.

4 Discours de Victoria Nulen: "From Dayton to Donetsk, Europe whole and free-", discours au "Croatia Summit", Dubrovnik, 11.7.2014. 
à l'extérieur et sa crise interne c'est l'inadéquation entre les objectifs et les moyens de la Politique européenne de voisinage (PEV) inaugurée il y a une décennie et les réalités d'aujourd'hui. Un triple changement est intervenu depuis.

D'abord, l'Europe a changé. Avec la crise économique et financière qui déboucha sur celle de l'euro, I'UE découvrait de nouvelles lignes de clivage Nord/Sud et la nécessité de redéfinir les conditions de sa cohésion interne. II se trouve que les pays du Sud de l'Europe, les plus touchés par la crise, sont aussi les vecteurs principaux de l'influence de l'UE sur la rive Sud de la Méditerranée. Transformée par son élargissement à l'Est qui a doublé le nombre d'Etats-membres et déplacé son centre de gravité vers I'Est, l'UE se devait d'élaborer une nouvelle approche de ses périphéries.

Les grands acteurs internationaux dans le voisinage européen, à commencer par la Russie et les Etats-Unis, ont, eux aussi, changé. La présidence Obama a amorcé un désengagement en Europe accompagnant son "pivot vers l'Asie" dans ses priorités stratégiques. Des Ełats-Unis sans doute moins présents dans le voisinage de l'Europe, mais toujours indispensables pour sa sécurité surtout, mais pas uniquement, aux yeux des nouveaux membres est-européens de I'UE et de l'OTAN. La Russie cherche à rétablir son statut de puissance internationale et sa sphère d'influence dans l'espace postsoviétique au prix de passer, au cours de la dernière décennie, d'une lente dégradation à une nouvelle "guerre froide" avec les Occidentaux.

Enfin, les voisinages de l'Union européenne ont profondément changé depuis l'élaboration première de la Politique européenne de voisinage dans le document "Wider Europe" de 2003: les Printemps arabes de 2011-2012, d'une part, et la révolution ukrainienne de 20132014, d'autre part, illustrent à leur manière, ces transformations: "wider Europe" rime désormais avec "wilder Europe" et voisinage avec insécurité. Entre l'Est et le Sud, la Turquie, qui en 2005 recevait le feu vert pour ouvrir des négociations d'adhésion à l'Union européenne, a montré depuis un durcissement de son régime à l'intérieur, de nouvelles ambitions à l'extérieur et un enlisement de sa relation avec l'UE. Seuls les Balkans poursuivent lentement et discrètement leurs processus de stabilisation dans une "perspective européenne". 
L'Europe est aujourd'hui exposée à une double déstabilisation de ses voisinages: à l'Est avec le conflit ukrainien qui bouleverse sa relation avec la Russie; au Sud avec un Moyen Orient passé du Printemps arabe de la démocratie à l'automne des généraux et à la déstabilisation de la région par l'implosion successive de la Libye, de la Syrie et de l'Irak qui ramènent les questions de sécurité et de migrations au premier plan de la relation avec l'Europe. Celle-ci se trouve prise simultanément sur ses deux flancs, Est et Sud, même si les Européens n'attachent pas tous la même importance aux deux voisinages et ne font pas la même lecture des enjeux qu'ils représentent. L'UE $a$, après de longues tergiversations, adopté des sanctions face à la Russie dans le conflit ukrainien5; elle resta complètement inaudible et inerte entre mai et juillet 2014 devant l'avancée de l'Ełat Islamique massacrant et expulsant les populations chrétiennes et la minorité kurdophone des yazidi, instaurant par la terreur un califat à cheval sur la Syrie et l'Irak. Malgré les efforts de la France pour "européaniser" ses engagements en Afrique, l'Allemagne d'Angela Merkel et la Grande Bretagne de David Cameron ne considèrent pas la poussée djihadiste dans la zone du Sahel comme un problème européen et voient le Mali ou le Centre Afrique comme un pré-carré postcolonial français. Les nouveaux membres de l'UE d'Europe du Centre-Est sont pour, des raisons historiques et géographiques, principalement préoccupés par leur voisinage oriental et le réveil de la Russie, alors que les pays du Sud de l'Europe subissent en premier les conséquences de la déstabilisation au Sud de la Méditerranée. En France, on est persuadé que pour la Pologne l'objectif "Ukraine et refouler la Russie" prime sur tout; en Pologne, on pense que la France préfère s'impliquer en Afrique Centrale plutôt qu'en Europe Centrale.

A côté des différences Est/Sud, analysées à travers des études de cas dans ce livre, on constate certains points de comparaison ou des problématiques communes: les aspirations et les limites de la démocratisation, les héritages des empires et l'Europe entre centre et périphéries.

1. Au Sud comme à l'Est de l'Europe on a assisté à l'effondrement soudain de dictatures ou de régimes semi-autoritaires par des mou-

5 L'UE a adopté le 29.7.2014 les mesures de "niveau 3" à l'égard de la Russie concernant les secteurs bancaire, l'énergie, la défense et les technologies sensibles. J-P. Stroobants, "Les nouvelles sanctions des Vingt-Huit visent des pans entiers de l'économie russe", Le Monde, 31.7.2014 
vements de contestation dont les places Tahrir au Caire et Maïdan à Kiev sont devenues les lieux emblématiques. Par delà la réflexion sur la fragilité des régimes despotiques ou, pour paraphraser JeanFrançois Revel, "comment les dictatures finissent"6, il y a d'abord l'irruption de la question démocratique et, plus généralement, des expériences contrastées des transitions qui ne mènent pas nécessairement à la démocratie. Certains on vu dans les Printemps arabes la sortie de l'exceptionnalisme arabe -qui dans le regard occidental ne serait qu'une variante de "I'orientalisme" (selon le terme d' Edouard Saïd) - et la confirmation de l'adhésion universelle aux valeurs démocratiques. II serait plus juste, sans doute, de parler d'une aspiration partagée à la dignité et à la liberté, mais ce n'est que la conquête de celles-ci qui permet ensuite de définir dans le processus de transition les valeurs et les principes sur lesquelles peut se fonder un nouvel ordre politique. Avec pour question centrale: comment construire un nouveau système politique capable de gérer la diversité religieuse (chiites/sunnites) qui représente à la fois un enjeu démocratique, mais aussi géopolitique (Iran/Arabie Saoudite)?

2. La chute des dictatures révèle la diversité des aspirations démocratiques, mais aussi la fragilité des Ełats, ceux nés de la décomposition de l'empire ottoman comme ceux nés, plus récemment, de l'éclatement de l'empire soviétique. Derrière la "sortie de Yalta" et l'ordre européen hérité de la Deuxième guerre mondiale, se profilait la fin de celui de Versailles et de la carte de l'Europe établie au lendemain de la première guerre mondiale. On le voit au Moyen Orient, comme à l'Est de Europe: plus de Yougoslavie, plus de Tchécoslovaquie, plus d'Union soviétique. De nouveaux Ełats-nations sont en gestation tant dans les Balkans qu'en Europe de l'Est révélant en cours des fractures anciennes et leur vulnérabilité au contexte régional.

Les nouvelles relations centre-périphérie en Europe sont tributaires de la décomposition d'un ordre étatique issu de celle des empires. Les "révolutions de couleur" en 2003-2004 en Géorgie, en Ukraine (et en

6 Jean-François Revel, Comment les démocraties finissent, Paris, Grasset, 1983. Le livre, publié quelques années avant la chute des dictatures communistes, commence par cette phrase:"La démocratie aura peut-être été dans l'histoire un accident, une brève parenthèse, qui, sous nos yeux se referme". 
Moldavie en 2009) avaient amorcé des transitions démocratiques, mais ont connu dans un deuxième temps des rechutes accompagnées d'une exploitation par Moscou de "conflits gelés" et de contentieux anciens sur les frontières et le statut d'enclaves, pour exercer une pression et tenter de ramener le pays dans l'orbite de la Russie. L'Union soviétique était à la fin de XXe siècle l'ultime avatar d'un empire colonial et la "reconquête" de la Crimée, comme la satellisation de l'Ossétie du Sud ou de la Transnistrie moldave sont les symptômes d'une situation post-impériale ou postcoloniale.

On retrouve, toute proportion gardée, des questions analogues dans le voisinage Sud. Les tentatives de démocratisation dans le monde arabe, imposées de l'extérieur par la force (intervention américaine en Iraq en 2003) ou impulsées de l'intérieur par les sociétés (Printemps arabe 2011), ont précipité la faillite des Etats accompagnée de guerres civiles. Derrière le "changement de régime" se profile l'écroulement des Etats (Libye, Syrie, Irak) et la fragmentation des sociétés dont la fragilité remonte à leurs origines c'est à dire à la façon dont les puissances européennes ont artificiellement découpé les dépouilles de l'Empire ottoman. On peut attribuer l'implosion de l'Irak en 2014 aux conséquences de la guerre que George Bush et certains alliés européens menèrent en 2003 pour imposer par le haut un "changement de régime". On peut aussi attribuer celle de la Syrie à un dictateur qui préfère la guerre et une partition implicite du pays pour sauver son régime face à une révolte populaire déclenché par le bas dans le sillage des Printemps arabes. Mais on peut aussi l'interpréter comme l'agonie d'une carte de la région établie par les Britanniques et les Français avec l'accord Sykes-Picot de 1916 et les traités de la fin de la première guerre mondiale, qui couvraient les anciennes provinces ottomanes allant de la Méditerranée au Golfe (Irak, Syrie, Liban,)7. Ainsi les grands bouleversements de l'après-Printemps arabe renvoient les Européens à leur propre rôle dans la fin des empires, les aventures postcoloniales et le retour du "grand jeu" dont ils sont largement éclipsés.

De nouveaux acteurs apparaissent, au premier rang desquels la Chine. D'anciens font leur retour avec lesquels l'Europe devra composer faute de les intégrer dans la PEV. La Russie comme la Turquie sont toutes deux héritières d'anciens empires; elles étaient le centre et non une périphérie.

7 Jolyon Ho worth, "Europe, Iraq and the end of Sykes-Picot", European Geostrategy, 18 juin 2014. 
Les impliquer dans une "politique européenne de voisinage" présuppose une improbable mutation de leur identité et des rapports avec leurs anciennes dépendances. II n'est pas évident de les impliquer dans une politique de voisinage européenne basée sur l'exportation d'un modèle normatif, le commerce avec l'UE, le dépassement des logiques de souveraineté et de puissance.

\section{Magnet Europa, I'UE comme "puissance transformatrice"}

La politique de voisinage de l'UE s'est largement inspirée de celle qui avait fait ses preuves dans le processus d'élargissement et qui donna naissance à la notion de "puissance transformatrice" de l'UE ${ }^{8}$. Cet "apprentissage institutionnel" est un classique étudié par les spécialistes de l'UE. Mais le modèle était-il transposable dans un environnement très différent, dans les voisinages de l'UE où dominent quatre thèmes: la démocratie, la sécurité, l'énergie, les migrations?

Le terme "élargissement" de l'UE pouvait donner l'impression ou l'illusion qu'elle resterait la même en plus grand. C'est en réalité une "autre Europe" qui se développe depuis 2004. Le terme de "réunification" européenne avait la préférence de Vaclav Havel et Bronislaw Geremek, les intellectuels d'Europe centrale qui ont incarné ce projet, car il suggérait des apports mutuels dans ces retrouvailles?. Pourtant, le discours sur la "réunification" n'était pas entièrement convaincant. D'abord, l'Europe n'était pas unie avant la guerre froide et surtout, au moment même où l'on signait solennellement le traité d'adhésion à l'UE des nouveaux membres à Athènes en avril 2003, elle étalait au grand jour sa désunion quant à la guerre américaine en Irak, opposant, selon la terminologie rumsfeldienne, la "vieille Europe" à la "nouvelle Europe". II est sans doute préférable de parler simplement d'un processus d'intégration européenne qui transforme les nouveaux membres de l'UE autant que l'Union elle-même.

8 L'une des premières à utiliser le terme fut Heather Grabbe, EU's Transformative Power, New York, Palgrave, 2006

9 Bronislaw Geremek, "De l'élargissement à la réunification. Qu'allons-nous apporter à l'Europe", in J. Rupnik (dir.) Les 
Mais, par delà les malentendus et les récits décalés il y a, dix ans après, la réalité d'un bilan qui les relativise et surtout explique pourquoi I'Union européenne et la perspective même lointaine de son élargissement garde un tel attrait sur sa périphérie. La "Grande Transformation" des vingt dernières années et dont l'intégration européenne fut l'aiguillon et la destination, comporte deux dimensions complémentaires qui ont un impact majeur sur la perception de I'UE dans son voisinage: la convergence spectaculaire des économies et des sociétés avec celles des anciens pays membres de l'UE, la consolidation de la démocratie et de l'Etat de droit. L'Europe agit ici comme "puissance structurelle" au sens que donne à ce terme Susan Strange, à savoir la capacité d'imposer sa logique économique, sécuritaire et politique à son environnement./ Note/

L'Elargissement n'est pas populaire dans les opinions publiques de I'Union. Près du $3 / 4$ des Autrichiens et des Allemands, plus de $2 / 3$ des Français, des Hollandais et des Belges, mais aussi 53\% des Tchèques n'y sont pas favorables 10. D'où le silence des élites politiques sur le sujet et leur embarras devant un drapeau européen agité à Kiev. La "fatigue de l'élargissement" souvent invoquée n'est qu'un aspect de la fatigue de l'Europe.

Mais si l'on veut comprendre la force de l'attrait que la perspective européenne continue d'exercer sur sa périphérie il faut, par delà la question de l'ancrage démocratique qui sera abordée, prendre en compte le succès spectaculaire de l'élargissement à l'Est de l'UE que résume le constat suivant: les vingt dernières années représentent pour l'Europe du Centre-Est une période historiquement sans précédent de modernisation et de convergence rapide avec l'Europe occidentale. Cette convergence des économies, des institutions, des modes de consommation, de l'espérance de vie, est directement liée au processus d'intégration européenne. C'est un immense succès de l'UE (qui évite d'en faire état) et devient à son tour un "aimant européen" pour sa périphérie.

10 Standard Eurobarometer 78 (automne 2012, T71). La question concernait la poursuite de "I'élargissement à d'autres pays dans les années à venir". 
$\Omega$ Convergence Est-Ouest du PIB /habitant au sein de I'UE

\begin{tabular}{|l|l|l|l|}
\hline & 2000 & 2005 & 2011 \\
\hline Austria & 132 & 125 & 129 \\
\hline Germany & 118 & 116 & 121 \\
\hline EU 27 & 100 & 100 & 100 \\
\hline Slovenia & 80 & 87 & 84 \\
\hline Czech Rep. & 71 & 79 & 80 \\
\hline Greece & 84 & 91 & 79 \\
\hline Portugal & 81 & 79 & 77 \\
\hline Slovakia & 50 & 60 & 73 \\
\hline Hungary & 54 & 63 & 66 \\
\hline Poland & 48 & 51 & 49 \\
\hline Romania & 26 & 35 & \\
\hline Bulgaria & & & \\
\hline
\end{tabular}

Source: Eurostat

Ce tableau de la convergence Est-Ouest au sein de l'UE doit être lu en parallèle avec celui de la divergence Est-Est. L'Ukraine avait il y a un quart de siècle, à l'époque du socialisme finissant, un PNB par habitant presque comparable à celui de la Pologne voisine. II est aujourd'hui trois fois inférieur 11. Entre 1990 et 2012 l'économie ukrainienne a perdu 30\%, celle de la Pologne a doublé. Les exportations ukrainiennes pendant la même période ont chuté de $40 \%$ alors que celles de la Pologne ont été multipliées par six. L'explication des trajectoires divergentes des deux pays (et plus généralement entre l'Europe centrale et l'Europe de 
l'Est)? : Transition démocratique, réformes structurelles et l'intégration européenne pour la Pologne; une pseudo-réforme, une économie et la politique dominée par les oligarques dans l'orbite de la Russie pour l'Ukraine.12 || serait erroné de ne voir derrière l'élan pro-européen des Ukrainiens au cours de l'hiver 2013-2014, des motivations principalement économiques, mais l'on ne peut occulter ces données de base.En effet, le rapprochement avec l'Union européenne est perçu dans son voisinage comme le moyen le plus sûr pour un pays de se moderniser et sortir de la pauvreté. Les pays du voisinage oriental (Ukraine, Moldavie, Géorgie, Arménie) sont les pays les plus pauvres d'Europe. Même la Bulgarie, pays le plus pauvre au sein de l'UE, a fait de rapides progrès depuis le feu vert donné à sa "perspective européenne" en 2000. Son revenu par tête est aujourd'hui le double de celui de l'Ukraine. II n'y a aucune gratitude envers l'Union pour ce rattrapage dans les nouveaux pays membres de I'UE, au mieux de l'indifférence comme l'ont montré les récentes élections européennes avec des taux de participation de 14\% en Slovaquie ou $18 \%$ en République tchèque. Mais le fort attrait qu'elle exerce sur son voisinage oriental ou dans les Balkans n'est pas lié à l'espoir de rejoindre l'euro, mais à la remarquable réussite dans l'UE des pays d'Europe centrale.

Différence de revenu par tête: UE-Balkans-Voisinage Est $^{13}$ (en \$ US)

\begin{tabular}{|l|l|l|}
\hline Pays & $\mathbf{2 0 1 2}$ & $\%$ \\
\hline UE (28) & 33,641 & 100.00 \\
Turquie & 10,830 & 32.19 \\
Bulgarie & 6,840 & 20.33 \\
Macédoine & 4,620 & 13.73 \\
Kosovo & 3,600 & 10.70 \\
Ukraine & 3,500 & 10.40 \\
Géorgie & 3,270 & 9.72 \\
Moldavie & 2,070 & 6.15 \\
& & \\
\hline
\end{tabular}

12 Pour une comparaison détaillée cf. Simon Tilford, "Poland and Ukraine: A tale of two economies", CER Bulletin n 95 (avril/mai 2014), Londres, Center for European Reform.

13 Source World Bank,, GNI per capita, atlas method (en \$ US). Tableau du revenu intérieur brut par tête, d'après une méthodologie établie par le prof. John Kay de l'Université d'Oxford, repris ici à partir du rapport de European Stability Initiative, "Why they Look West- Ukraine, Poverty and the EU" (mars 2014) http://www.esiweb.org/ rumeliobserver/2014/03/20/why-they-look-west-ukraine-poverty-and-the-eu/ 


\section{L'UE et la diffusion de la démocratie}

La seconde dimension majeure de l'attrait de l'UE concerne la démocratie et l'Etat de droit. L'Europe n'affiche pas, à la différence des Etats-Unis, une doctrine de "promotion de la démocratie" et encore moins de "mission démocratique"14. Elle a pourtant apporté une contribution décisive à la consolidation de la démocratie en Europe du Centre-est. Son programme méconnu de diffusion de la démocratie s'appelle "I'élargissement" de l'Union européenne et c'est certainement le plus grand succès de l'UE des deux dernières décennies. L'élargissement aux pays du Sud de l'Europe sortant de la dictature (Espagne, Portugal, Grèce) avait implicitement fait de l'UE un ancrage pour les transitions à la démocratie. Mais on peut dire que l'élargissement à l'Est à amené l'UE à se définir plus explicitement comme une union de démocraties qui, dans sa relations extérieures, à commencer par son voisinage, inclue le soutien à la démocratisation. Son levier principal relève de ce qu'Adenauer appelait "Magnet Europa": le désir d'appartenance à l'UE, c'est I' "aimant européen", qui amène les élites politiques des Etats candidats à adopter des normes et des institutions compatibles avec celles de l'UE. C'est une intégration asymétrique pas une vraie négociation) quirepose sur la motivation et les intérêts des prétendants, nul recours à la force; l'inverse de celle préconisée par l'administration Bush. La pire menace que pouvait professer l'UE c'est: "nous n'allons pas vous envahir!", c'est-à-dire vous inclure dans l'Union. La perspective européenne créait dans les années 90 pour les élites politiques centre-est européennes une situation où le coût politique et économique de la non-intégration devenait supérieur au coût non négligeable de l'intégration. C'est ce levier qui a prévalu même en Slovaquie avec une coalition pro-européenne opposée la dérive nationaliste et autoritaire du régime Meciar, avec lliescu et les postcommunistes en Roumanie, le parti socialiste en Bulgarie autour des années 2000 ou, plus récemment, le HDZ croate sous la direction d'Ivo Sanader, héritier de Franjo Tudjman. Cela n'exige pas une "conversion" aux valeurs européennes, mais une forte dose de réalisme et une volonté

14 Sur l'évolution de l'approche américaine sous les présidences Bush et Obama, voir Thomas Carothers, in J.Rupnik (ed.) 1989 as a Political World Event, Democracy, Europe and the New International System in the Age of Globlisation, Londres, Routledge, 2013, p 72-79. Pour la contribution européenne cf. Marieke van Doorn et Roel von Meijenfeldt (dir.), Democracy Europe's Core Value, on the European profile in world-wide democracy assistance, Delft (P-B), Eburon Academic Publishers, 2007; + discours de V.Havel au lancement de la European Foundation for Democracy through Partnership (EPD) à Bruxelles le 15 avril 2008 en présence du président de la Commission européenne, cf aussi le discours de V.Havel en ouverture du colloque "1989 as a global event", CERI-Sciences Po le 23.10.2009. 
politique prête à froisser quelques intérêts acquis. II n'a évidemment pas de prise sur des élites politiques (Meciar, Milosevic, Loukachenko ${ }^{15}$ ) qui, à juste titre, comprennent les implications de la contrainte européenne pour leurs régimes. Cette intégration asymétrique fut en Europe centrale d'une grande efficacité dans la fabrication d'un consensus nécessaire (et provisoire) pour les réformes structurelles de l'après-1989.

Dans quelle mesure cette logique des transferts normatifs et institutionnels à l'oeuvre dans le processus d'élargissement de I'UE peut-elle rester pertinente pour la Politique européenne de voisinage (PEV) ? La spécificité de l'approche européenne ce n'est pas de préparer un "changement de régime", mais la consolidation d'une transition démocratique, la gouvernance plus que la démocratie, la construction d'un Etat de droit à travers le processus d'adhésion centré sur l'adoption de normes et d'institutions capables de les appliquer c'est à dire garantir les droits des citoyens et autonomie des acteurs économiques et sociaux: justice indépendante, administration publique non-partisane, décentralisation, lutte contre la corruption. La "puissance transformatrice" de l'UE opère par deux biais: la conditionnalité, selon les critères de Copenhague adoptés par l'UE en juin 1993, et la socialisation des acteurs impliqués dans le long processus d'adhésion à I'UE. Dans les études qui lui sont consacrées les " transferts normatifs " de l'UE vers ses périphéries sont improprement appelés "européanisation", une version nouvelle, certes revue et corrigée, de la "mission civilisatrice" de l'Europe. ${ }^{16}$

En réalité, entre l'émetteur de normes (UE) et les récepteurs une dialectique s'instaure qui combine un degré d'imposition (il s'agit bien d'une intégration asymétrique) et une dose d'adaptation par les acteurs et leurs pratiques ${ }^{17}$. Le second élément est décisif pour l'appropriation et donc les effets durables du processus. Une adoption formelle de normes sans modifier les comportements ne permet pas réellement l'établissement

15 Sur le régime Loukachenko et les raisons internes de son ancrage à l'Est cf. Valeri Karbalevitch, Le Satrape de Biélorussie, Paris, François Bourin Editeur, 2012 avec une introduction de Stéphane Chmelewski, ancien Ambassadeur de France en Biélorussie.

16 Pour une lecture critique du discours et l'UE et, dans son sillage, de nombreux spécialistes des études européennes cf Jan Zielonka, "Europe's new civilizing missions: the EU's normative power discourse", Journal of Political Ideologies (n 1 / 2013) p 35-55

17 Une des premières élaborations de ce thème F. Schimmelfenig, "The Impact of International Organisations on Central and East European States- Conceptual and Theoretical Issues" in R.Linden (ed.) Norms and Nanies, Langham (Md.), Rowman \& Littlefield, 2002; Sur la place du 'levier' européen dans les transitions cf. Milada Vachudova, Europe Undivided: democracy, leverage and integration, Oxford, Oxford University Press, 2005 cf aussi Thierry Delpeuch, "Questions de recherche", CERI, 2013 
d'un Etat de droit et s'apparente pour certaines adhésions récentes à une "européanisation Potemkine"18.

Le transfert institutionnel et normatif s'accompagne de transferts considérables d'ordre financier dans la phase de pré-adhésion, mais surtout après: pour un pays comme la Pologne cela représente plus de 100 milliards d'euros pour la période 2007-2013, montant en légère augmentation pour la période budgétaire en cours qui va jusqu'en 2020. Cet ancrage externe pour la transition à la démocratie et à l'économie de marché fut très efficace en Europe centrale et peut être considéré comme l'apport européen majeur à la diffusion de la démocratie. Une seule ombre au tableau: ce levier de la démocratisation s'avère beaucoup moins efficace après l'adhésion à l'UE comme l'a récemment démontré la régression dans certains pays, particulièrement de la Hongrie de Viktor Orban. ${ }^{19}$

La PEV avait pour but d'utiliser une "boîte à outil" largement dérivée de celle établie pour l'élargissement ${ }^{20}$, avec cependant deux différences majeures: les protagonistes, les pays concernés par le Partenariat oriental sont différents (état de la démocratie, niveav économique, élites politiques et culture politique) de ceux d'Europe centrale et surtout la finalité n'est pas la même. Le but du processus d'adhésion à l'UE est, selon la formule du ministre des affaires étrangères polonais, de se "trouver une place à la table plutôt que sur le menu". Si la PEV ne peut offrir cette perspective de quels leviers dispose-t-elle?

La politique européenne de voisinage fut conçue pour accompagner le grand l'élargissement à l'Est à dix nouveau pays membres le 1 mai 200421. Elle avait pour objectif de "partager avec les pays limitrophes les avantages de l'élargissement de l'UE de 2004 - c'est-à-dire la stabilité, la sécurité et la prospérité-dans les conditions distinctes d'une adhésion à l'UE. Elle vise

18 Tom Gallagher, Romania and the EU, how the weak conquered the strong, Manchester, Manchester University Press, 2009

19 Jacques Rupnik, "Régression post-démocratique en Hongrie", Esprit (avril 2013); et "La crise de la démocratie en Europe Centrale et Orientale", in Dominique Reynié (dir.) L'Union européenne en 2014, Paris, Lignes de Repères, 2013, p 15-24. Cf aussi Veselin Ganev, "Post-accession hooliganismu in East European Politics and Society, n 1 /2013

20 Pour une présentation détaillée de la PEV et de ses instruments cf. notre chapitre "L'Europe et ses périphéries" in J.Rupnik, Les Banlieues de l'Europe, les politiques de voisinage de l'Union européenne, Presses de Sciences Po, 2007 ainsi que le chapitre de Pierre Mirel dans ce volume.

21 Le 1 mai 2004 marque l'adhésion à l'UE de dix pays: Pologne, Hongrie, République tchèque, Slovaquie, Slovénie, de trois pays baltes Estonie, Lettonie, Lituanie ainsi que Chypre et Malte. En janvier 2007 s'y ajoutèrent la Roumanie et la Bulgarie, puis, en juillet 2013, la Croatie. 
à prévenir l'apparition de nouvelles lignes de fracture entre l'Union et ses voisins et offrir à ces derniers l'occasion de participer à diverses activités de I'UE par le biais d'une coopération étroite sur les plans politique, économique et culturel, ainsi qu'en matière de sécurité"22. Günter Verheugen, alors Commissaire chargé de l'élargissement, précisait que les pays du voisinage "ne bénéficient pas d'une perspective d'adhésion future". ${ }^{23}$ Autrement dit: éviter de dresser de nouvelles barrières avec les pays du voisinage et, en même temps, proposer une relation qui ne passe pas par la perspective d'une future adhésion. Le président de la Commission européenne Romano Prodi résumait alors la philosophie de la politique de voisinage: construire autour de l'UE un "cercle d'amis" auxquels on proposait une association étroite à l'UE sans la perspective d'une adhésion, "tout sauf les institutions". ${ }^{24}$

\section{Diversification Est-Sud de la PEV}

La mise en œuvre de cette politique au cours de la décennie suivante amena au cours de la dernière décennie par touches successives des rectifications et des redéfinitions concernant tant la diversité des voisinages que la finalité de la PEV. Rappelons que la PEV, comprenant aujourd'hui 16 pays ( 6 à l'Est et 10 du Sud), devait, selon le rapport initial élaboré par Chris Patten et Javier Solana en 2002, comprendre trois catégories de pays: les pays du Sud de la Méditerranée, les pays des Balkans, enfin la Russie et "les autres voisins de l'Est". ${ }^{25}$ La Russie s'est vite récusée, se voyant mal dans la même catégorie d'interlocuteurs que la Moldavie ou l'Azerbaïdjan, et préférant un "partenariat privilégié" avec l'UE. Mais c'est surtout sous l'impact des "révolutions de couleurs", particulièrement de la "révolution orange" en Ukraine en décembre 2004, qu'il devenait difficile

22 Communication de la Commission IP/04/632, "Après l'élargissement: la Commission donne un coup d'accélérateur de la politique européenne de voisinage", Bruxelles, 12 mai 2004

Cité par Thomas Ferenczi, "L'Union veut offrir un 'partenariat privilégié' à ses nouveaux voisins", Le Monde, 15 mai 2004.

24 Pour une analyse de la genèse de la PEV cf. notre chapitre introductif in J.Rupnik, Les Banlieues de l'Europe, les politiques de voisinage de I'Union européenne, Paris, Presses de Sciences Po, 2007, p 9-40. Une analyse plus détaillée de l'élargissement à l'Est et de la PEV est présentée dans ce volume dans le chapitre de Pierre Mirel. 
de mettre sous la même rubrique "voisinage" des pays du Moyen Orient et d'Europe de l'Est. Une différentiation dans la PEV s'esquissa entre les "voisins européens" à l'Est et les "voisins de l'Europe" au Sud. Les premiers, soutenus par les nouveaux membres centre-européens de l'UE, souhaitant bénéficier d'une perspective d'adhésion qui ne pouvait être offerte aux seconds. Sans que l'UE fasse sienne la "perspective européenne" pour les voisins de l'Est on note en 2005 un glissement dans le discours de Javier Solana, Haut Représentant pour la politique étrangère et de sécurité de l'UE : "La politique européenne de voisinage n'est pas destinée à l'élargissement de I'UE; elle ne le prévoit ni ne l'interdit". ${ }^{26}$ Jusqu'où peut-on cultiver l'ambiguitée? Dans sa présentation du bilan de la PEV devant le Parlement européen à la veille du Printemps arabe, Stefan Fülle, le Commissaire à l'élargissement qui supervise la mise en œuvre de la PEV, fit sienne la formule suivantesur son objectif à long terme qu'il situait "entre la zone de libre échange approfondie (DCFTA) + $1 \%$ et l'adhésion à l'UE -1\%". ${ }^{27}$

Le véritable tournant dans la différenciation eut lieu en 2008. A la veille du lancement, à l'initiative de la France qui exerçait alors la présidence de I'UE, d'une Union pour la Méditerranée en juillet 2008, la Pologne et Suède présentèrent en mai un mémorandum préconisant un "partenariat oriental" de l'UE pour les pays d'Europe de l'Est post-soviétique. La différentiation de la PEV correspondait sans doute à une nécessité (comment proposer et mettre en œuvre une approche commune pour l'Ukraine et la Jordanie?), mais aussi à des agendas concurrentiels au sein de l'UE entre les pays du Sud et de l'Est considérant leurs voisinages respectifs comme prioritaire pour l'engagement politique de l'UE et les moyens financiers afférant (1/3 à l'Est, 2/3 au Sud). C'est ainsi que l'on assista à quelques mois d'intervalle au lancement à Paris de l'Union pour la Méditerranée en juillet 2009 et celui du Partenariat oriental en avril 2009 à Prague sous la présidence tchèque de l'UE. Les deux connurent, pour des raisons différentes, une mise en œuvre difficile.

Le projet de l' "Union pour la Méditerranée" se justifiait par la nécessité de donner un nouvel élan à la politique de voisinage Sud après une décennie d'un "Processus de Barcelone" à bout de souffle. Josip Borell, président

26 Javier Solana interrogé par Celia Chaufour, CAUCAZ.COM, 4.11.2005.

27 Commissionner S.Fülle, ENP Strategic Review, European Parliament Foreign Affairs Committee, Bruxelles, 26 octobre 
"barcelonais" du Parlement européen affirmait alors: "Je dois dire qu'en dix années le processus de Barcelone ne nous a pas mené bien loin sur la voie vers un espace commun de paix de prospérité"28. Quand on lit les discours prononcés en 2005 à l'occasion de son dixième anniversaire à Barcelone, où aucun des leaders du Sud ne fit une apparition (!), on ne peut que constater des résultats extrêmement modestes dans les trois domaines principaux (les trois "corbeilles" serait-on tenté de dire en empruntant au langage du "processus de Helsinki" dans l'Europe divisée d'avant 1989) à savoir la sécurité, les échanges économiques et l'évolution politique et les droits de l'homme. Sur la sécurité, on n'osait plus parler d'un processus de paix israélo-palestinien autrement que pour regretter les occasions manquées dans la décennie précédente. Lors de la guerre du Liban de 2006, le Conseil européen n'arriva même pas à se mettre d'accord pour un appel au cessez-le feu. La menace terroriste liée à la radicalisation d'une partie de la mouvance islamiste restait une préoccupation majeure comme l'ont rappelé les attentats de Madrid. Même constat décevant sur l'objectif de créer une zone de libreéchange méditerranéenne prévue pour l'an 2000 et surtout le constat que l'écart de niveau de vie entre les deux rives de la Méditerranée au lieu de se réduire s'était accentué au cours de la décennie écoulée. Enfin, n'ayant pas réussi à favoriser une démocratisation des régimes au Sud on se contenta de prôner une amélioration du respect des droits de l'homme et une meilleure prise en compte de la société civile.

Si un concept politique ne donne pas les résultats escomptés on est en droit de préconiser une approche renouvelée. Eł c'est ainsi que fut présenté le lancement, par la présidence française de I'UE, de "I'Union pour la Méditerranée". Au delà de l'étrangeté du nom (quelqu'un serait-il "contre" la Méditerranée ?) plusieurs interrogations et objections sont vite apparues. Elles peuvent se résumer ainsi: avec qui et sans qui? Cette fois les leaders du Sud, de Mubarak à Assad, étaient bien présents aux côtés de président Sarkozy lors du sommet de Paris le 13 juillet 2008, indiquant par la même que la "troisième corbeille" de la démocratisation ne serait pas vraiment une priorité de UpM. Leur présence contrastait avec le

28 Josip Borell à Barcelone le 28 novembre 2005: "Malgré trois milliards d'euros alloués par les fonds MEDA la frontière de l'Europe avec la Méditerranée reste la plus inégalitaire au monde. Aucune autre n'a de différences de revenus aussi importantes avec une telle proximité. ...En dix ans le PNB par tête des Quinze s'est accru de 50\%, passant de 20000 à 30000 dollars. Pour les dix nouveaux membres de l'Union il est passé de 6000 à 15000 dollars. Pendant la même période, au Sud de la Méditerranée, le revenu par tête a stagné entre un peu moins de 5000 dollars à un peu plus de 5000 dollars". www.europarl.europa.eu/ former_ep_presidents/president -borell/speeches/en/files. 
grand absent, à savoir l'Union européenne. Car dans le projet, lancé par la présidence française de l'UE, on n'avait pas fait de place pour les instances européennes: étaient concernés les pays méditerranéens de I'UE, pas l'Union en tant que telle. L'objection de l'Allemagne à cette régionalisation de l'UE amena, dans un second temps, une modification du concept initial en ramenant l'UE dans le jeu, mais le mal était fait: Berlin et les pays du Nord furent d'emblée réticents au projet; ceux de l'Est se consolèrent vite de voir un lancement problématique pour le "voisinage concurrent". Le partenaire privilégié dans la conception française de I'UpM n'était pas la Commission, mais la Turquie. Cela était sensé présenter un double avantage: un pont avec le monde islamique sur la rive Sud de la Méditerranée et un substitut à la perspective d'adhésion de la Turquie à I'UE. La Turquie a bien compris le message et préféra jover sa propre carte au Sud de la Méditerranée.

Le Printemps arabe de 2011 aurait pu être l'occasion pour UpM de trouver un second souffle, une nouvelle raison d'être. II n'en fut rien entre les hésitations de la diplomatie française et les admonestations de Catherine Ashton sur la nécessité de promouvoir une "démocratie profonde" qui ne se limiterait pas aux élections libres. Le lancement de l'UpM sans la mettre clairement sous le toit de l'Union européenne s'avéra être un handicap politique majeur même si ce sont bien les pays du Sud européen qui sont les plus directement en prise avec les changements sur l'autre rive de la Méditerranée. Or, au moment du Printemps arabe ils se trouvaient aussi être les plus durement touchés par la crise économique et financière de la zone euro et par conséquent en mauvaise position pour soutenir le changement et impulser un engagement européen plus conséquent. L'Allemagne et les pays du Nord furent prompts en 2008 à contrer l'initiative méditerranéenne française la jugeant, à juste titre, insuffisamment européenne. Ils ne firent pas grand chose ensuite pour proposer une autre politique européenne (et des moyens correspondants) en phase avec le Printemps arabe.

En réponse au Printemps arabe, l'Union européenne s'efforça de définir un cadre renouvelé pour sa politique de voisinage avec trois volets, trois " $M$ " (money, market, mobility): mise en place d'une zone de libre échange, des mesures favorisant une meilleure circulation des personnes (problème des réadmissions), et une aide financière accrue selon un 
programme de soutien aux réformes basé sur la conditionnalité connu sous le vocable"more for more": plus d'aide européenne pour plus de réformes de la gouvernance dans les pays du Sud. ${ }^{29}$

Les échecs du printemps arabe ne sont pas attribuables aux politiques européennes. Force est de constater que des progrès relatifs au Maghreb (surtout en Tunisie et dans une moindre mesure au Maroc) semblent plus liés à des dynamiques internes accompagnée par l'interaction avec certains pays de la rive Nord de la Méditerranée, tandis qu'au Moyen Orient, où prévalent les considérations de sécurité (Syrie, Irak, Libye, conflit israélo-palestinien), I'UE est toujours éclipsée par d'autres acteurs internationaux. Résultat: on assiste, après le Printemps, au retour en force des deux préoccupations majeures de l'UE face à son voisinage Sud: la sécurité et les migrations.

La sécurité concerne les répercussions en Europe des conflits sur le continent africain (de la Syrie au Mali) entre la poussée jihadiste et la menace terroriste en Europe. La question des migrations est redevenue un enjeu majeur de la PEV et de la vie politique dans la plupart des Etats de I'UE. Son analyse doit partir d'un double constat: un mythe et une absence.

L'idée d'une "forteresse Europe" est un mythe en train de devenir une politique. La "forteresse" est combattue par les associations humanitaires soucieuses de défendre le droit d'asile et du regroupement familial ainsi que les partisans d'une "Europe sans barrières" (devise de la présidence tchèque de l'UE en 2009) prônant une ouverture maximum au commerce et à la mobilité. Elle est aussi une illusion dangereuse chez ceux qui pensent que la fermeture européenne à l'âge de la mondialisation reste une option..$^{30}$ Depuis plus de vingt ans, l'Europe accueille chaque année plus d'un million et demi de migrants légaux ce qui est plus que les terres d'immigration par excellence que sont les Etats-Unis, le Canada, l'Australie et la Nouvelle Zélande réunies. La "forteresse" rhétorique et

29 La réponse européenne aux Printemps arabes est développée dans les chapitres de P. Mirel et A. de Vasconcelos ainsi que dans la conclusion par Gilles Kepel.

30 Pour replacer les problèmes de migration européens dans un contexte global voir les travaux de Catherine Witold de Wenden, cf."Les flux migratoires légaux et illégaux", CERISCOPE Frontières, 2011 
règlementaire fut et reste en porte à faux avec les réalités tandis qu'une "tentation du mur" commence à se faire jour dans un contexte de crise économique et de défaillance de la politique européenne.

Deuxième constat:I'UE a aboli les frontières en son sein et garantit la liberté de circulation et d'installation à ses citoyens, mais ne s'est pas dotée d'une politique migratoire européenne alors que les réalités démographiques et économiques au Sud autant que l'insécurité et l'effondrement des Ełats accentuent la pression migratoire extra-communautaire. Le risque c'est que l'objectif de restreindre celle-ci amène (comme on l'observe aujourd'hui en Grande Bretagne face à l'émigration d'Europe de l'Est) des pressions pour restreindre les libertés d'installation ou de travail au sein de l'UE. On observe donc une interaction entre les migrations intracommunautaires de l'Est et celles, extra-communautaires, du Sud qui instaure la confusion entre la difficile gestion des flux migratoires et la libre circulation au sein de l'UE qui est l'une des quatre libertés fondatrices de celle-ci.

La Turquie a, ces dernières années, revu à la baisse sa coopération concernant le contrôle des flux migratoires venus d'Afghanistan et du Moyen Orient avec des répercussions immédiates pour la Grèce, pressée à son tour par les pays de la zone Schengen de mieux contrôler sa frontière. La Bulgarie, devenue pays de transit, s'est lancé dans la construction sur trente kilomètres d'un mur haut de trois mètres à la frontière avec la Turquie $^{31}$. Il y a vingt-cinq ans, après la chute du régime communiste, le rideau de fer entre les deux pays construit pour empêcher les Bulgares de sortir avait été démantelé. Aujourd'hui un mur est construit pour empêcher les migrants d'entrer en Bulgarie, c'est à dire dans I'UE...

Lampedusa, Malte, Patras ou Ceuta et Melilla sont devenues aux yeux des Européens les symboles d'un drame humanitaire, mais aussi de l'incapacité des Etats à contrôler leurs frontières et de l'absence d'une politique européenne coordonnée. L'impuissance des pays européens comme de l'UE à réguler les flux migratoires fait écho dans les opinions publiques à celle dont ils font preuve face à la mondialisation ou la crise financière. L'argument démographique des experts de la Commission concernant la complémentarité à la

31 Elise Vincent, "L'Europe s'emmure pour repousser les migrants", Le Monde, 11 novembre 2013. 
longue entre la jeunesse des populations du Sud et le vieillissement de celles du Nord semble faire consensus, mais n'est que partiellement convaincant. Selon le sociologue Johan Wets "L'Ouest demandera surtout des travailleurs hautement qualifiés alors que l'offre du Sud est surtout composée de personnels faiblement ou non-qualifiés." 32

Autrefois on pouvait distinguer entre les nationalistes anti-immigration et les anti-européens. Aujourd'hui ce sont les mêmes, les deux ressorts se renforcent mutuellement et leur percée aux élections européennes de mai 2014 devrait être un avertissement pour l'UE. Cela ne pourra que renforcer à court terme le manque évident de solidarité parmi les VingtHuit lorsqu' il s'agit du contrôle des frontières ou de l'accueil des réfugiés ${ }^{33}$. L'idée d'un Commissaire européen aux migrations coordonnant une étroite collaboration de Frontex avec le Service d'Action Extérieur et les responsables de la politique de voisinage serait un moyen de combiner les volets humanitaire, sécuritaire et diplomatique de cette politique. La capacité à formuler une politique migratoire européenne dans son ensemble et dans la durée, à cogérer et réguler les mobilités sera un enjeu politique majeur de la décennie à venir au même titre que l'euro ou la sécurité ${ }^{34}$. Retour à la dialectique entre des frontières de l'Europe ef de la nature des frontières en Europe.

\section{Voisinage Est: les limites du partenariat oriental}

Le Partenariat Oriental inauguré à Prague en avril 2009 proposait une politique de voisinage spécifique destinée à l'Europe de l'Est postsoviétique avec le triptyque: accord de libre-échange "approfondi et complet" qui suppose une adoption par les pays concernés de normes européennes (politique de la concurrence qui bloque les aides de l'Etat, normes phytosanitaires au nom de la protection du consommateur) en échange d'un accès au marché de l'UE. Ensuite, il s'agissait de faciliter la mobilité des citoyens par une libéralisation progressive de la politique de visas. Enfin, des aides financières ciblées ont été mises en place pour accompagner les réformes.

32 Johan Wets (Université de Louvain), Les Nouvelles Migrations, un enjeu européen, Bruxelles, Editions Complexe, 2013.

33 La France, l'Allemagne et la Suède ont absorbé en 2013 57\% des demandeurs d'asile au sein de l'UE. Cf. Jean Pierre Stroobants, "Le Commissaire européen à la migration déjà abandonné?", Le Monde, 17 juillet 2014.

34 Pour une proposition d'un cadre supranational pour gérer les zones d'interdépendance migratoires intenses entre les Européens et les pays de départ cf. Hubert Védrine, "Pour une gouvernance véritable des migrations", Le Monde, 24 mai 2011. 
Le Partenariat Oriental fut promu au nom d'une nécessaire différentiation Est-Sud de la politique de voisinage. On découvrit cependant très vite la diversité au sein même de ce groupe d'Etats postsoviétiques. Certains ont des frontières avec l'UE (Belarus, Ukraine, Moldavie) ce qui n'est pas le cas pour les trois pays du Caucase (Géorgie, Arménie, Azerbaïdjan). La plupart ont des contentieux territoriaux ou des "conflits gelés": la Géorgie avec les enclaves d'Ossétie du Sud et d'Abkhazie, l'Arménie est en conflit avec son voisin l'Azerbaïdjan depuis un quart de siècle pour le Haut-Karabakh (enclave arménienne en territoire azéri où un cessez-le feu fut négocié avec la médiation de la Russie en 1994 et rompu vingt ans après) ${ }^{35}$, la Moldavie avec une Transnistrie qui fait sécession, et, plus récemment, I'Ukraine dont une partie du territoire, la Crimée, fut annexée par la Russie en mars 2014. Le seul partenaire sans problème territorial, la Biélorussie, reste aussi le plus improbable étant donné la nature de son régime politique. Avec ces contentieux territoriaux le voisinage oriental reste une zone d'instabilité -grande différence avec l'Europe centrale après 1989et l'UE a, hormis les encouragements d'usage et la philosophie générale de son projet ("le commerce adoucit les mœurs"), peu d'influence sur leur issue."Le Partenariat Oriental n'est pas un mécanisme de résolution des conflits" a déclaré le Commissaire en charge du Partenariat. ${ }^{36}$ || pourrait, en favorisant médiations et coopération régionale, devenir un mécanisme de prévention des conflits ${ }^{37}$. L'UE offre un cadre de coopération, mais n'est pas un acteur majeur de la sécurité dans un voisinage sous influence d'un autre acteur de poids : la Russie. Cet engagement ambigu de l'EU sur sa périphérie peut s'avérer être une arme à double tranchant: plus on s'avance vers l'Est (ou le Sud), plus l'UE se trouve impliquée dans des zones d'instabilité ou de conflits déclarés sans avoir les moyens d'y faire face ou sans toujours pleinement mesurer les conséquences pour elle.

Le deuxième facteur de différentiation parmi les pays du Partenariat Oriental, c'est leur régime politique et leur orientation entre l'UE et la Russie. Sur plus de deux décennies on peut distinguer dans le monde postcommuniste trois grandes trajectoires des transitions. En Europe centrale

35 Joshua Kucera, "Bloody clashes between Azerbaijan and Armenia aver disputed territory" EurasiaNet, The Guardian, 4. 8. 2014 Le conflit éclata lors de la dissolution de l'URSS faisant 30000 morts et un million de personnes déplacées. La vulnérabilité de cette enclave arménienne en territoire azéri est l'une des raisons majeures des relations étroites de l'Arménie avec Moscou, principal garant de sa sécurité face à son voisin plus riche et mieux armé.

37 B.Coppitiers et al., Europeanisation and conflict resolution: case studies from the European periphery, Gent, Academia 
on est passé de la transition à la consolidation de la démocratie (une élection à Prague ou à Varsovie est un enjeu de gouvernement et non de régime) et la construction (très imparfaite) d'un Etat de droit compatible avec l'appartenance à l'UE.

Dans les Balkans la transition à la démocratie fut dévoyée par le primat de la question nationale, c'est du cadre territorial contesté dans lequel s'inscrit de la nouvelle démocratie. La guerre dans l'ex-Yougoslavie, la question des frontières et des minorités nationales, favorisait l'union sacrée dans chacune des républiques plutôł que l'émergence du pluralisme politique. Ce n'est que depuis la fin de la guerre de dissolution de l'ancienne Yougoslavie que l'on observe simultanément la construction de nouveaux Etats-nation et d'institutions démocratiques. En cela il y a des parallèles avec les nouveaux Ełats dans l'espace post-soviétique. Avec une différence de taille: perspective, clairement affichée depuis le sommet de Salonique en juin 2003, d'intégration des Balkans occidentaux dans l'Union européenne.

Dans l'espace ex-soviétique les transitions ont donné naissance à des régimes hybrides que les politologues Levitsky et Way définirent comme "competitive authoritarianism"38, c'est à dire des régimes qui tolèrent un certain pluralisme politique et organisent des élections libres dont les résultats sont validés par l'OSCE, mais qui ne permettent pas sur une véritable compétition politique ouverte et "à armes égales". La séparation des pouvoirs reste une fiction et les média publics sous influence de l'exécutif. L'économie et la sphère politique (les partis, média) sont imbriqués et contrôlés par quelques oligarques liés au pouvoir. Ce pluralisme oligarchique post-soviétique était plus proche de celui qui prévaut en Russie que du modèle centre-européen. On peut le définir, selon la formule paradoxale de Marie Mendras à propos du régime de Poutine, comme "un pouvoir fort et un Etat faible"39. La verticale du pouvoir est la clef du politique, les institutions de l'Etat de droit sont atrophiées et surtout corrompues, l'espace de la société civiles restreint et étroitement

38 S.Levitsky- L.Way, Competitive Authoritarianism, hybrid regimes after the cold war, Cambridge, Cambridge University Press, 2010. 
C'est ici que la question du régime politique dans le voisinage oriental rejoint celle déjà abordée du modèle de développement économique et du contraste entre Europe de l'Est post-soviétique et Europe centrale. Selon Daron Acemoglu et James Robinson, la clef pour interpréter les différences entre les pays qui "réussissent" et ceux qui "échouent" dans les mutations de l'économie mondialisée réside précisément dans le caractère de leurs institutions ${ }^{40}$. Les premiers sont en général ceux qui ont des institutions "inclusives", garantissant les normes de l'Etat de droit, une distribution pluraliste du pouvoir favorisant l'initiative citoyenne et l'entreprenariat, les seconds adoptent un modèle politique etéconomique fondé sur l' "extraction": la concentration du pouvoir et l'extraction des ressources aux mains de quelques uns. A des degrés divers les pays du partenariat oriental correspondaient à ce diagnostic et ce n'est qu'au cours de la dernière décennie que l'on assista à d'importantes tentatives de changement.

\section{Les "révolutions de couleur" (suite)}

La "révolution des roses" en novembre 2003 en Géorgie et la "révolution orange" en décembre 2004 en Ukraine ont remis en question le modèle politique semi-autoritaire en place depuis l'éclatement de l'URSS. Elles furent parfois un peu hâtivement présentées comme un prolongement de 1989 avec cependant une différence importante notée par Vaclav Havel: "les révolutions de 1989 furent des révolutions contre le communisme" alors que les "révolutions de couleurs" furent des "révolutions contre le postcommunisme", que l'on peut définir comme un "mélange de régime autoritaire et de capitalisme mafieux"41.

L'Ukraine comme la Géorgie connurent au cours de la décennie suivante des reculs et des évolutions contrastées. Le président Saakashvilli en

40 Daron Acemoglu et James Robinson, Why Nations Fail, London, Profile Books, 2012. 
Géorgie fut critiqué pour la forte concentration du pouvoir aux mains de l'exécutif et pour son "aventurisme" dans l'opération de reconquête de l'Ossétie du Sud en 200842; on peut avoir juridiquement raison (reprise du contrôle sur des territoires faisant partie de la Géorgie) et politiquement tort (l'opération à haut risque fournit à la Russie un prétexte pour intervenir). Mais Saakashvilli a aussi, après ses deux mandats, accepté l'alternance en léguant un bilan dont l'acquis majeur reste un combat contre la corruption qui a apporté des résultats.

L'après 2004 avec éclatement de la "coalition orange" entre ses leaders entre louchtchenko et Timochenko en Ukraine et surtout la régression qui a suivi l'élection à la présidence en 2010 de Viktor lanoukovitch, leur adversaire de 2004, ont déçu les espoirs de démocratisation. Et l'on peut interpréter la révolution"Euromaidan" de 2013-2014 comme une manière de prolonger dans un contexte différent, celle, inachevée, de 2004. L'étincelle ce fut bien la non-signature le 28 novembre 2013 de l'Accord d'Association avec l'UE par le président lanoukovitch, mais c'est le rejet d'un système autoritaire et corrompu qui devint rapidement la cible principale du mouvement. L'invocation de l' "Europe" représentait tantôt une référence identitaire, tantôt une demande d'Ełat de droit et l'abolition de la kleptocratie. La deuxième phase du mouvement fut déclenchée en janvier 2014 par l'adoption par le pouvoir de mesures répressives. On passe alors de l'enjeu européen à un enjeu de régime, c'est à dire la résistance à la "poutinisation" du pouvoir ukrainien. C'est là que revint en force un élément déjà présent sous une forme moins radicale lors de la "révolution orange", le lien entre la question du régime et l'orientation géopolitique du pays entre l'Europe et la Russie.

\section{Le rôle des pays d'Europe Centrale dans le voisinage oriental}

42 L'analyse des correspondants du New York Times conteste la version officielle d'une opération " défensive " dans le déclenchement des bombardements de Tskhinvali où se trouvaient aussi des soldats russes stationnés là dans le cadre d'une opération de " maintien de la paix ". C.J. Chivers et Ellen Barry, " Georgia claims on Russia war called into question", New York Times, 6 novembre 2008. Pour une étude approfondie du conflit plus favorable au président géorgien et une analyse du role des Etats-Unis et de la mediation européenne cf. Ronald D. Asmus, A Little War that Shook the World, Georgia, Russia and the West, New York, Palgrave, 2010 
C'est sans doute cette dimension géopolitique et son potentiel explosif qui ne furent pas, semble-t-il, pleinement mesurés dans l'élaboration et la mise en œuvre par I'UE du Partenariat oriental et de l'Accord d'Association proposé au sommet de Vilnius en novembre 2013. Ceux qui au sein de l'UE mettaient d'emblée l'accent sur l'enjeu géopolitique du partenariat oriental étaient ses premiers promoteurs, les pays d'Europe centrale. Leur intérêt de sécurité paraît clair: stabiliser la périphérie orientale tout en refoulant l'influence russe dans cette zone. Un objectif secondaire, lié au précédent, était, en contribuant à façonner la PEV vers l'Est, de renforcer leur propre rôle au sein de I'UE ainsi que la dimension atlantiste puisque les Etats-Unis comptent sur ces alliés pour la "promotion de la démocratie" après les "révolutions de couleur". Les pays d'Europe centrale bénéficient d'atouts importants pour jouer ce rôle: la proximité, la connaissance des pays concernés, l'implication d'acteurs de la société civile concernée par le voisinage, enfin une légitimité liée à l'idée d'un "transfert d'expériences" tiré de leur propres transitions à la démocratie et à l'économie de marché après 1989.

Parmi les nouveaux membres de l'UE le rôle central revient à la Pologne, premier promoteur du Partenariat oriental. La Pologne a en effet, pour des raisons historiques, des liens privilégiés avec l'Ukraine et la Biélorussie. De 1569 jusqu'au partage de la Pologne à la fin du XVIllème siècle, l'Union Pologne-Lituanie comprenait une partie du territoire de l'Ukraine actuelle ainsi que la Biélorussie ${ }^{43}$. L'Ukraine occidentale (Lvov/Lviv) faisait partie de la Pologne jusqu'à la Deuxième guerre mondiale et le nationalisme ukrainien, fort dans cette partie du pays, était à la fois anti-russe et anti-polonais. La Pologne a su, au lendemain de l'éclatement de l'URSS, dissiper les appréhensions que pouvait susciter son engagement dans son voisinage en étant la première à reconnaître l' indépendance des nouveaux Ełats postsoviétiques et cultivant de bonnes relations avec Kiev indépendamment de la couleur politique du gouvernement. A côté des ONG internationales (principalement américaines) c'est la Pologne qui apporta le principal soutien à la "révolution orange" de 2004. Son dénouement fut facilité par la médiation à Kiev en pleine crise du trio européen composé du Haut Représentant, Javier Solana, accompagné des président polonais et lituanien. L'UE ne se serait sans doute pas autant engagée dans la crise ukrainienne sans la pression polonaise. La Pologne, qui venait d'adhérer à

43 Daniel Beauvois, Les confins de l'ancienne Pologne, Lille, Septentrion, 1995. Cf. aussi Pouvoir russe et noblesse polonaise en Ukraine 1793-1830, Paris, CNRS, 2003. 
I'Union en mai 2004, n'aurait jamais eu l'opportunité de jouer en décembre ce rôle de médiateur en Ukraine sans le parapluie de l'UE.

Dix ans après, l'Ostpolitik des pays d'Europe Centrale doit être réévalué à l'aune de la crise ukrainienne de 2014. Le Groupe de Visegrad ("V4" Pologne, Hongrie, République tchèque, Slovaquie) et les pays Baltes, est devenu le principal promoteur du Partenariat Oriental au sein de l'UE et les sommets de Prague (2009) sous la présidence tchèque de l'UE, celui de Budapest (2011) sous la présidence hongroise et finalement celui de Vilnius (2013) sous la présidence lituanienne confortèrent cette image. Elle est en partie conforme à la volonté de certains pays d'Europe centrale de s'impliquer à travers le partenariat oriental dans le transfert institutionnel et normatif vers les voisins de l'Est. ${ }^{44}$

Il convient cependant de nuancer cette présentation pour plusieurs raisons. D'abord un engagement dans le voisinage oriental pouvait très bien être divorcé d'un souci de l'Union Européenne. Les jumeaux Kaczynski, leaders du courant national-conservateur au pouvoir entre 2005 et 2007, étaient à la fois hostiles à l'intégration européenne (trop dominée par l'Allemagne) et réclamaient un engagement fort de I'UE dans le voisinage de l'Est (trop dominé par la Russie). L'ancien président tchèque Vaclav Klaus, europhobe notoire qui aime comparer l'UE à une "nouvelle URSS", recommandait néanmoins son élargissement à la Turquie et même au Kazakhstan. Le président Roumain Traian Basescu, qui préconisait au moment de l'adhésion de son pays à l'UE la formation d'un "axe stratégique Washington-Londres-Bucarest", n'a pas hésité, à l'occasion de l'ouverture du sommet de Vilnius sur le Partenariat Oriental le 28 novembre 2013, de recommander une méthode nouvelle pour l'adhésion de la Moldavie à l'UE: son annexion par la Roumanie! On ose espérer qu'un référendum serait organisé.

La crise ukrainienne fut un test majeur - sans doute le plus important depuis 1989 - de la pertinence de la coopération centre européenne. On pouvait, en effet, supposer qu'une révolution dans le pays le plus important du voisinage oriental doublée d'une menace de sécurité russe allait démontrer la cohésion et l'utilité du groupe de Visegrad. II n'en

44 Elsa Tulmets, "Les transferts d'expérience de l'Europe centrale et orientale vers le voisinage européen: rhétorique ou réalité? Les cas polonais et tchèques", Les études du CERI, 2013. V4 et EP, IIR 
fut rien, chaque pays réagissant différemment, selon des intérêts et des préoccupations divergents, démontrant que ces pays ne sont pas un bloc homogène au sein de I'UE comme on les présente parfois (atlantistes, libéraux en économie et favorable à tout ce qui pourrait refouler l'influence russe de leur voisinage). La Pologne joua dès le début un rôle actif dans la réponse à la crise ukrainienne tant au plan européen (médiation à Kiev de la troïka des ministres des affaires étrangères français, allemand et polonais le 21 février 2014) qu'au niveau de l'OTAN (augmentation de la présence militaire américaine en Pologne pour renforcer les garanties de sécurité des pays Baltes). Pendant la phase la plus dramatique de la crise le ministre des affaires étrangères polonais s'est rendu à Kiev avec ses homologues français et allemands pour tenter une médiation qui n'a pas abouti. Pour chercher une réponse à la crise ukrainienne on préfère le Groupe de Weimar (France, Allemagne, Pologne) plutôt que le Groupe de Visegrad.

Ce n'était pas vraiment surprenant étant donnée l'attitude indulgente envers la politique russe en Ukraine adoptée par la Hongrie. En présence à Bratislava de ses collègues du V4, le premier ministre Viktor Orban considéra les régimes russe et ukrainien aussi peu démocratique I'un que l'autre. II a, par ailleurs, signé en 2014 avec Moscou un accord pour la construction de deux nouveaux réacteurs nucléaires. Eł surtout: sans justifier l'annexion russe de la Crimée (au nom de la protection des populations russophones) Viktor Orban a demandé au gouvernement de Kiev une autonomie et des garanties pour la protection de la minorité hongroise en Ukraine (estimée à un quart de million d'habitants) ${ }^{45}$.

A mi-chemin entre la position polonaise et celle de la Hongrie on trouve celles de la République tchèque et de la Slovaquie: condamnation de l'annexion de la Crimée, prudence dans la relation avec Moscou au nom de considérations commerciales et refus catégorique de tout déploiement de troupes de l'OTAN dans les pays membres de l'alliance. Au moment où, donnant suite aux appels de la Pologne et des pays baltes, le président Obama demandait au Congrès de débloquer un milliard de dollars supplémentaire pour augmenter la présence militaire américaine en Europe de l'Est le premier ministre tchèque déclarait à Vienne le 4 juin

45 La confrontation sur l'Ukraine fut vive entre les premiers ministres hongrois et polonais lors de la conférence Globsec à Bratislava à la mi-mai 2014. Orban ajouta lors d'un entretien télévisé que l'on trouvait au sein de l'UE différents types d'autonomie parmi lesquels la minorité hongroise d'Ukraine pourrait choisir. E† tout gouvernement ukrainien doit être conscient que la Hongrie soutiendra les demandes de la minorité hongroise. Le moment est approprié puisque l'Ukraine est en reconstruction. Cité par MartinEhl, "Playing with Fire", Transitions online 20.5.2014. 
2014 que la République tchèque "ne soutient pas le renforcement de la présence militaire de l'OTAN en Europe" ${ }^{46}$. Son homologue slovaque Robert Fico alla jusqu'à comparer un éventuel déploiement de forces de l'OTAN dan son pays à l'invasion soviétique de 1968...

Pendant la dernière décennie le groupe de Visegrad (V4) gardait un profil bas et semblait uni par un soutien tacite au Partenariat oriental de I'UE. La crise ukrainienne a fait voler en éclats cette unité de façade. "L'Ukraine aurait pu placer le V4 sur la carte géopolitique. Au lieu de cela elle l'a effacé". ${ }^{47}$

Tableau: Evaluation de l'Etat de la démocratie dans l'espace postcommuniste ${ }^{48}$

\begin{tabular}{|l|l|l|l|}
\hline Slovénie & 1.89 & Bosnie & 4.39 \\
\hline Estonie & 1.96 & Ukraine & 4.86 \\
\hline Lettonie & 2.07 & Géorgie & 4.75 \\
\hline Hongrie & 2.89 & Moldavie & 4.82 \\
\hline Slovaquie & 2.57 & Arménie & 5.36 \\
\hline République tchèque & 2.14 & Kosovo & 5.25 \\
\hline Lituanie & 2.32 & Kirghizstan & 5.96 \\
\hline Pologne & 2.18 & Russie & 6.21 \\
\hline Bulgarie & 2.89 & Tadjikistan & 6.25 \\
\hline Roumanie & 3.50 & Azerbaïdjan & 6.64 \\
\hline Serbie & 3.64 & Kazakhstan & 6.57 \\
\hline Croatie & 3.61 & Belarus & 6.71 \\
\hline Macédoine & 3.93 & Ouzbékistan & 6.93 \\
\hline Monténégro & 3.82 & Turkménistan & 6.93 \\
\hline Albanie & 4.25 & & \\
\hline
\end{tabular}

L'Europe et le retour de la géopolitique

46 Bohuslav Sobotka, premier ministre, lors de sa visite en Autriche, cité par El5 (Prague) 9.6.2014. L'ancien président Vaclav Klaus, conservateur-libéral, exprima sa compréhension pour l'annexion de la Crimée au nom d'arguments historiques et politiques. Cf A. Illarionov et D. Rohac "Former President Klaus" Flawed Defence of Crimea's Annexation" in World Affairs (Cato Institute) www.worldaffairsjournal.org 24.7.2014

47 Selon Edward Lucas, correspondant est-européen de The Economist, "V4: Grappling with irrelevance", Central European Policy Institute (www.cepolicy.org) 30.6.2014; cf aussi M.Ehl, "Will the Visegrad Group Survive Ukraine" Transitions online, 17.6. 2014 entendu trop précises pour être "vraies". Elles donnent cependant une indication étant donné les critères pris en compte et surtout doivent être pris en compte sur une période donnée. L'important pour notre propos est de voir que les trois groupes de pays (Europe Centrale, Balkans, Europe de l'Est post-soviétique) restent distincts sur deux décennies. 


\section{Sécurité, énergie et géopolitique}

L'expérience de la construction européenne théorisée par la science politique dans l'approche des voisinages repose sur la notion d'interdépendance, de réseaux de communication et de coopération qui permettent de désamorcer les conflits à commencer par la façon même dont ils sont énoncés. Prolongeant cette optique fonctionnaliste de la paix par l'intégration et la rationalité des intérêts des acteurs certains adeptes de l'école "constructiviste" privilégient l'étude des discours par rapport au poids des "structures matérielles" dans l'explication ou la recherche de la causalité des conflits. Dans cette approche discursive "le conflit existe si les acteurs articulent leur incompatibilités mutuelles. Si celles-ci ont une base matérielle ou pas ne nous intéresse pas pour notre diagnostic du conflit. En fait, dans la mesure où les structures matérielles sont elles-mêmes construites par le discours, la différentiation entre les structures et le discours n'a pas de sens". ${ }^{49}$ Autrement dit ce sont les processus d'intégration ou d'association à l'UE qui peuvent contribuer à transformer les modes de communication et les pratiques discursives qui construisent le conflit. Au-delà des apports de l'approche fonctionnaliste (désamorcer les conflits par l'interdépendance) dérivée de l'histoire de la construction européenne et de l'approche "discursive" qui considère les conflits d'intérêts et d'identités comme socialement construits, force est de constater qu'elles ont une capacité explicative faible dès que l'on aborde les enjeux, les limites et les échecs récents des politiques européennes de voisinage. D'autres approches peuvent s'avérer pertinentes quand on parle de menaces de sécurité, de puissance ou d'enjeux énergétiques pour l'Europe, bref de géopolitique dans son voisinage ${ }^{50}$.

L'approche géopolitique des relations internationales se fonde sur l'idée que les choix de politique étrangère (économiques et de sécurité), sont façonnés par la position géographique d'un Ełat, sa taille, sa population et les ressources dont il dispose ${ }^{51}$. Dans la théorie réaliste la compétition

49 Thomas Diez, Mathias Albert et Stephan Stetter, The European Union and Border Conflicts, the power of integration and association, Cambridge, Cambridge University Press, 2008, p 7.

50 Cf Christopher Hill, "The geopolitical implications of enlargement" in Jan Zielonka (ed.) Europe Unbound, enlarging and reshaping the boundaries of the European Union, 2002, p 95-116.

51 Pour une étude très complète des origines et usages du concept cf. Claude Raffestein, Dario Lopreno, Yvan Pasteur, Géopolitique et Histoire, Paris, Payot, 1995. Pour une critique féroce du concept cf l'ouvrage du fondateur de la revue Hérodote, Yves Lacoste, La géographie, ça sert, d'abord, à faire la guerre, 1976, nouvelle édition, Paris, La Découverte, 2012. 
pour les ressources, l'énergie aujourd'hui en premier lieu, devient un enjeu géostratégique majeur. "L'énergie est à la fois un facteur qui influence les résultats de la politique étrangère des Etats et un instrument potentiel de leur politique étrangère". ${ }^{2}$ Entre ressources énergétiques et la politique il s'agit d'une interaction plutôt qu'une détermination. Les Européens, relativement dépourvus de ressources énergétiques, dépendent de leurs voisinages Est et Sud richement dotés, mais politiquement instables et imprévisibles, sont à la recherche d'une stratégie énergétique européenne dans le cadre d'une gouvernance mondiale de l'énergie. ${ }^{53}$

Depuis la crise pétrolière de 1973 le problème pour l'approvisionnement de l'Europe était au Sud et la solution à l'Est. C'est alors que furent amorcés les premiers échanges gaziers entre la Russie et les pays de l'Union européenne qui n'ont cessé de se développer depuis. Aujourd'hui la crise ukrainienne fait surgir des craintes concernant la dépendance envers la Russie. D'où la question: puisque l'Europe craint l'incertitude dans l'approvisionnement de l'Est, la solution pourrait-elle venir du Sud?

Si la quête de fiabilité est une motivation première il n'est pas évident que I'Iran, I'Irak ou la Libye représentent une alternative. Le voisinage Sud est politiquement encore plus instable que celui de l'Est. II existe cependant un développement qui pourrait modifier la donne à savoir la découverte récente en Méditerranée orientale, entre Israël et Chypre, de gisements considérables de gaz. Cette découverte, "change la donne géopolitique" selon le président turc et a des implications politiques régionales considérables pour la relation entre Israël, Chypre et la Grèce. ${ }^{54}$ Et l'on peut envisager une modification de la donne politique entre ces trois pays et les relations tendues de chacun d'entre eux avec la Turquie. Mais comme le note fort justement Michael Leigh la causalité tend à être inverse: "le progrès politique dans la résolution des conflits est nécessaire pour que les peuples de la région bénéficient d'un niveau optimum de coopération dans le développement des ressources énergétique de la région"55. Autrement dit, ce ne sont pas les pipelines qui produisent la

52 Brenda Shafer, Energy Politics, Philadelphia, University of Pennsylvania Press, 2009, p. 28

53 Sur la gouvernance globale de l'énergie cf. C. Kéréble et J.H. Keppler (Dir.) La gouvernance mondiale de l'énergie, gouvernance européenne et géopolitique de l'énergie, Paris, IFRI, 2009 chap. 2, pp 37-120 et conclusion pp 249-256.

54 Pour les implications régionales de cette nouvelle donne énergétique cf. l'analyse de Michael Leigh, "Energy - A Geopolitical Game Changer" in The International Spectator, vol 49, n 2, juin 2014, p 1-10.

55 Michael leigh, "Energy a geopolitical game changer?", art.cit. p 9 
paix, c'est la paix qui peut créer les conditions pour un investissement dans les pipelines. En attendant la réponse à la question de l'interaction entre la gestion de ces nouvelles ressources énergétiques et la capacité à désamorcer les conflits dans la région (israelo-palestinien ou la partition greco-turque de Chypre) l'alternative du Sud à l'approvisionnement énergétique de l'UE en provenance de l'Est reste à l'état de projet.

La Russie et avant elle I'URSS a commencé à livrer du gaz sibérien à l'Allemagne depuis le début des années 1980 et s'est "toujours comporté comme un fournisseur fiable de I'Union européenne".56 Au cours des années 2000, avec l'élargissement à l'Est de l'UE, l'interdépendance énergétique s'est accentuée pour représenter près d'un tiers des importations gazières en Europe ${ }^{57}$. Cette dépendance envers la Russie est très inégalement répartie puisqu'elle est nulle pour l'Espagne et quasitotale pour les pays d'Europe Centrale (seule la Pologne et la République tchèque l'ont réduite au cours de la dernière décennie pour atteindre $2 / 3$ et de leurs besoins ${ }^{58}$ ). L'Allemagne reste le partenaire privilégié de la Russie depuis la mise en service du gazoduc Northstream en $2011 . "{ }^{59}$.

\section{/Insérer: Carte d'approvisionnement en gaz de l'UE/}

Cette situation s'est cependant altérée au cours de la dernière décennie avec la détérioration des relations entre la Russie et l'Ukraine et parallèlement avec I'UE. Selon la théorie des jeux appliquée au triangle Russie-Ukraine-UE on peut imaginer différents modèles de coopération ou de gestion de conflits et l'on est passé en une décennie par plusieurs

56 C. Kérébel, op.cit. p 85

57 Catherine Locatelli, "Les échanges gaziers entre l'UE et la Russie: des interdépendances aux incertitudes", Questions internationales (Documentation française) n65 (janvier-février 2014, p 60-62; V. Lavicka-M.Hudema "Rusky plyn deli Evropu" (le gaz russe divise l'Europe), Hospodarske Noviny (Prague) 26.6.2014.

58 Un exemple de politique à long terme: La République tchèque à commencé en 1994 la construction du pipeline Ingostadt qui permet de s'approvisionner en Allemagne et de Norvège qui fournit 1/3 des besoins tchèques. Par le système de "reversion" (la possibilité d'envoyer le gaz non seulement Est vers Ouest , mais aussi à l'inverse) la Tchèquie a pu fournir la Slovaquie pendant la crise du gaz en Ukraine au début 2009.

59 Cécile Kérébel, "Quelle gouvernance pour les marchés du pétrole et du gaz?" in Kérébel et Keppler (dir) La gouvernance mondiale de l'énergie, op.cit. p 85 
variantes. ${ }^{60}$ Un modèle de coopération où une logique de calcul rationnel prévaut dans la gestion des conflits d'intérêt au bénéfice des trois acteurs; un modèle où un des axes (Russie-Ukraine) est rompu et les deux protagonistes cherchent séparément la réassurance auprès du troisième (UE), mais qui peut aussi, troisième option, mener à la rupture du deuxième axe (UE-Russie). Entre calcul rationnel et la confiance qui ne l'est pas toujours c'est une illustration quasi parfaite du "dilemme du prisonnier" où la rupture de confiance amène les deux protagonistes à préférer une option perdante ${ }^{61}$. Plusieurs issues de sorties sont envisageables: La réconciliation et le retour au triangle initial. Ou, I'un des acteurs, se considérant en position de force, persuade le second de se dispenser ou de marginaliser - le troisième (c'est la logique de Northstream entre la Russie et l'Allemagne). Enfin, une variante qui consiste à introduire dans le jeu un nouvel acteur: la Turquie impliquée dans toutes les stratégies de contournement de la Russie comme de I'Ukraine; la Chine comme alternative à terme pour la Russie au marché européen ${ }^{62}$.

Une première alerte en janvier 2006 suivie d'une crise plus grave en janvier 2009 avec l'accord Putin-Tymochenko63 fit comprendre aux européens que la "guerre du gaz" russo-ukrainienne représentait aussi une menace pour eux. C'est dans la crise ukrainienne l'Europe découvrit le besoin d'options alternatives et d'une "stratégie énergétique"64. La première difficulté dans son élaboration résidait dans le degré de dépendance inégal envers la Russie et les différences d'approche de la relation UERussie. L'Allemagne avait avec North Stream, un gazoduc sous la mer Baltique, pour son approvisionnement de Russie. Le projet Nabucco avait pour but réduire la dépendance de l'approvisionnement russe en construisant un pipeline liant la mer Caspienne à l'Europe via les pays du Caucase et la Turquie. Près d'une décennie ans après sa conception les réalisations ne suivent pas et -à moins d'un sursaut politique et financierNabucco risque, selon la formule de ses détracteurs, de "rester un opéra".

60 Pour une approche dérivée de la théorie des jeux cf. Pavel K.Baev, "Energy Intrigues on the EU's Southern Flank, applying game theory", Problems of Post-Communism, vol 57 n 3 (May-June 2010) p 11-22

61 Selon le 'dilemme du prisonnier' en théorie des jeux formulé en 1950 par Albert Tucker chacun des deux joueurs préfère trahir l'autre car si l'un coopère et l'autre pas le coopérant est lourdement pénalisé. 23 mai 2014

63 L'accord qui fut par la suite reproché à Tymochenko prévoyait un paiement mensuel pour l'importation du gaz russe afin de remédier à l'accumulation de livraisons impayées, clause qui s'avéra intenable pour les finances de l'Ukraine qui demanda une remise, obtenue en 2010 à condition que la Russie reste le fournisseur exclusif. Cf Baev, art.cit p 11. 
Le projet South Stream vise, à l'inverse, à réduire la dépendance du transit par l'Ukraine par un gazoduc russe de près de mille km sous la Mer Noire vers la Bulgarie puis, via la Serbie, vers la Hongrie, la Slovénie et I'Italie avec un embranchement vers la Hongrie vers l'Autriche. Les principaux partenaires de Gazprom dans le projet sont la compagnie italienne ENI et la compagnie autrichienne ÖMV qui crée en juin 2014 une joint venture avec Gazprom. 16 milliards d'euro c'est le prix que le fournisseur et les pays clients sont prêts à payer pour le contournement de l'Ukraine. South Stream augmenterait sans doute la sécurité des livraisons, mais aussi la dépendance envers le fournisseur russe réduisant ainsi la marge de manœuvre des européens à un moment où un bras de fer politique et économique (sanctions) était engagé entre l'UE et la Russie concernant l'Ukraine.

C'est ce contexte qui a amené la Commission européenne à se rallier à la demande américaine et ukrainienne d'envoyer un signal à Moscou et arrêter South Stream. Le premier ministre ukrainien latseniouk a soumis en juin 2014 au parlement un projet de loi pour la vente de $49 \%$ du réseau ukrainien de pipelines aux occidentaux ce qui permettrait, selon lui, d'obtenir de meilleurs contrats de transit, mais aussi "d'arrêter la construction du gazoduc South Stream et acheminer le volume prévu pour ce nouveau pipeline par l'Ukraine". ${ }^{65}$ Sortir de la dépendance énergétique envers la Russie c'est aussi le message américain aux les Européens et c'est aux côtés du sénateur McCain que le premier ministre bulgare Plemen Orecharski annonça la suspension du projet South Stream ${ }^{66}$. L'Union européenne de son côté invoquait les règles de la concurrence non respectées par Gazprom et le Commissaire à l'énergie, Günther Oettinger, qui dans un premier temps récusait les conseils américains $s^{67}$ admettait cependant que la crise ukrainienne avait "ralenti considérablement le progrès de South Stream"68.

Avec Nabuco, North Stream et South Stream les Européens ont proposé trois réponses différentes à la question ukrainienne qui est aussi et surtout

65 Cité par Vladimir Divis, "Jistota za desitky miliard" (La sécurité pour des dizaines de milliards), Pravo (Prague), 26.6.2014

66 Stanley Reed, "A pipeline caught in the political crossfire", International New York Times, 2.7.2014

67 Le Commissaire allemand à l'énergie Günther Oettinger déclara en juin 2014: "C'est nous, les pays membres de l'UE et non les USA, qui achetons le gaz russe. Nous pouvons nous consulter avec les Etats-Unis. Nous écoutons volontiers leurs recommandations, mais les décisions nous appartiennent". cité par V.Divis, art.cit. 
une question russe et constitue un enjeu géopolitique majeur. D'où la recherche d'une stratégie énergétique européenne proposée par la présidence polonaise de l'UE en 2009 (investir dans les infrastructures qui permettront l'interconnexion des réseaux européens, possibilités d'acheter du gaz liquéfié aux Ełats-Unis, développer de nouvelles ressources telles que le gaz de schiste). La crise ukrainienne de 2014 a accéléré l'élaboration de ces projets pour aboutir à la proposition franco-polonaise d'une"communauté européenne de l'énergie" visant à convaincre l'ensemble des pays membres d'adopter une stratégie qui rende l'Europe "plus indépendante, plus cohérente, plus solidaire dans sa politique énergétique "69. Bien qu'ayant le soutien de la présidence du Conseil européen la réponse qui importe est moins à Bruxelles qu'à Berlin: l'Allemagne ayant décidé sa "sortie du nucléaire" aura encore moins l'intérêt à remettre en question son "partenariat énergétique privilégié" avec la Russie.

C'est par rapport à ces enjeux qu'il faut évaluer l'impacte possible des sanctions à l'encontre de la Russie adoptées par l'UE en juillet 2014 visant les secteurs de l'énergie, des technologies "sensibles" civiles et militaires et la capacité de la Russie de se financer sur les marchés européens alors que le pays connaît une fuite massive des capitaux (75 milliard au premier semestre de 2014, le double de l'année 2013)..$^{70}$ La Russie riposta par des sanctions concernant l'importation de produits alimentaires et en haussant le prix du gaz: premier avertissement aux Européens concernant le recours possible, en cas d'escalade, à l'arme énergétique. ${ }^{71}$ C'est là que l'interdépendance entre l'UE et la Russie est la plus forte et c'est elle qui fait de la crise ukrainienne un enjeu qui la dépasse: la question russe de l'Europe.

La "révolution orange" de 2004 provoqua dans la politique russe un choc, un tournant comparable, selon Ivan Krastev, au 11 septembre 2001 pour la politique américaine. Ce tournant, ayant une dimension externe (affectant les relations avec l'Europe et les occidentaux) et interne (le durcissement autoritaire et nationaliste du régime) fut parachevé

69 Présentation par le président français et le premier ministre polonais Donald Tusk à Paris, l'Express le 24.4.2014. Le projet fut proposé au sommet européen du 27 juin 2014. Cf aussi. Albert Bressand, Transition énergétique européenne: bonnes intentions et mauvais calculs, Paris, Fondapol, 2013

70 J-P. Stroobants, "Les nouvelles sanctions des Vingt-Huit visent des pans entiers de l'économie russe", Le Monde 31.7.2014

71 Marie Jégo, "La Russie brandit l'arme du gaz en réponse aux sanctions", Le Monde, 1.8.2014 
pendant la révolution "Euromaidan" de Kiev dix ans plus tard. L'Ukraine comme catalyseur au cours de la dernière décennie de la redéfinition de l'identité russe et de la relation entre la Russie et l'Occident ${ }^{72}$. Le ciment idéologique de ce tournant c'est la résurgence d'un nationalisme postimpérial dont on peut identifier quatre éléments principaux.

1. La réaffirmation par la Russie comme "grande puissance" dans un monde multipolaire qui "vise à contenir l'hégémonie américaine" et se caractérise, selon le politologue russe Igor Zevelev, par un discours sur la "préservation de la souveraineté de la nation par tous les moyens et d'empêcher toute forme d'ingérence dans les affaires de l'Ełat"73. Le discours sur la modernisation économique a été remplacé par la géopolitique et l'affirmation du primat de la puissance de l'Etat s'est traduite par une augmentation du budget militaire de $100 \%$ en dix ans et une capacité à se positionner comme interlocuteur indispensable sur de grands dossiers internationaux tels que le nucléaire iranien ou la crise syrienne. Depuis le milieu des années quatre-vingt avec l'arrivée au pouvoir de Gorbatchev jusqu'à la fin de l'ère Eltsyne (et même les premières années de Poutine) la Russie se considérait comme appartenant à la "Grande Europe" et menait une politique étrangère assez convergente avec celle des occidentaux. Ce "suivisme" est depuis dénoncé comme ayant été exploité par les Occidentaux pour affaiblir et marginaliser la Russie et étendre I'OTAN à ses frontières. ${ }^{74}$

2. Du temps de l'Union soviétique on distinguait dans des cycles longs de "guerre froide" (avec de courtes périodes de détente) et de longues périodes de détente (avec des rechutes courtes de tension). Avec Gorbatchev puis Eltsyne on a connu une période sans précédent dans l'histoire russe de réformes et de pluralisme, d'ouverture interne et externe sur l'Europe-certes entrecoupé de tensions ponctuelles. Après une période de détérioration depuis une décennie la Russie

72 Pour une analyse du lien entre la redéfinition de l'identité russe et la stratégie européenne et internationale de la Russie à partir de la présentation de la nouvelle doctrine stratégique par le Conseil des Affaires Internationales de Russie cf. la conférence de Zbigniew Brzezinski, "Mutual Security on Hold? Russia, the West and the European Security Architecture ", Wilson Center, Washington, 16 juin 2014. Le rapport du Conseil Russe pour les Affaires internationales de 2014 s'articule autour de quatre notions: "un peuple divisé"; "protections des compatriotes à l'étranger"; un "monde russe" (Roussiki mir), la promotion de la "grande civilisation russe". 
est entrée avec la crise ukrainienne de 2013-2014 dans un cycle long de nouvelle "guerre froide". Celle ci n'est pas, bien entendu, la continuation de l'ancienne: il n'y a pas d'ambition planétaire soutenue par une idéologie. Mais elle se caractérise par une nouveauté importante: alors qu'auparavant la Russie distinguait entre l'OTAN, sa bête noire qui représente une menace et l'UE qui était considérée essentiellement comme un ensemble économique et commercial (pour la politique la Russie préférait s'adresser aux grands Etats-membres). On s'opposant à la signature par Kiev e l'Accord d'Association Moscou indiquait clairement qu'elle rangeait désormais l'UE dans la même catégorie que I'OTAN.

3. Cette nouvelle posture vis à vis de l'Europe s'accompagne de l'affirmation que la Russie et le "monde russe" sont porteurs d'une "civilisation singulière", ni occidentale ni asiatique, qui repose sur des valeurs chrétiennes et une "mission historique": défendre le valeurs traditionnelles face à un occident matérialiste et décadent (l'UE comme propagateur du mariage gay). On retrouve là, bien entendu, les ingrédients anciens des débats du XIXe siècle entre slavophiles et occidentalistes et surtout le courant "eurasien", influent dans l'émigration russe des années vingt et auquel se rattache explicitement l'un des idéologues du régime poutine Alexander Dougine ${ }^{75}$. Une version russe du "choc des civilisations" où la Russie se pose en s'opposant.

4. L'élément sans doute le plus important et le plus déstabilisant dans la nouvelle doctrine impériale russe, énoncée par Poutine le 18 mars 2014 selon laquelle "le peuple russe est devenu le plus grand peuple dispersé dans le monde". D'où il n'y a qu'un pas vers l'idée de rassemblement des populations russes et russophones comme grand projet géopolitique neo-impérial pour la Russie. D'où le mélangé dans le nouveau discours en vigueur à Moscou de retour aux "fondamentaux" de l'empire russe d'antan et de volonté de retrouver son influence dans "I'étranger proche" où vivent vingt-cina millions de russes/russophones.

A la différence d'autres empires coloniaux construits "outre mer", l'empire

75 A Doulgine, entretiens avec Alain de Benoît

cf Pour une tentative d'établir un lien entre la redécouverte de l'idéologie eurasienne et le projet d'Union eurasienne proposé par Poutine cf Alexander Lukin, art.cit. Foreign Affairs, July-August 2014 
russe s'est constitué par une extension progressive au fil des siècles de ses frontières. Après l'indépendance de l'Inde la Grande Bretagne restait la Grande Bretagne; après l'indépendance de l'Algérie, la France restait la France. Mais personne à Moscou n'a fourni une réponse à la question qu'estce que la Russie sans empire, quelles sont ses frontières?. Le problème avec la Russie, selon Vaclav Havel, c'est qu' "elle ne sait pas où elle commence et où elle s'arrête". La réponse que donne le nouveau cours nationaliste à Moscou c'est la Russie est partout où il y a des Russes. Réponse dangereuse puisqu'elle amena, au nom de la "protection des Russes", au rattachement de la Crimée à la Russie: première annexion d'un territoire en Europe depuis 1945 et violation du principe d'inviolabilité des frontières selon les accord de Helsinki de 1975, confirmé par la Charte de Paris de 1990.

Pour justifier sa démarche la Russie invoque le précédent yougoslave: le Kosovo qui, suite à l'intervention militaire de l'OTAN de 1999 et un référendum, s'est détaché de la Serbie. Trois différences essentielles invalident le parallèle: l'indépendance du Kosovo fut rendue possible par une décennie "d'apartheid" sous Milosevic alors que personne ne menaçait les Russes de Crimée. Le Kosovo est indépendant, mais non rattaché à une Grande Albanie (comme la Crimée à la Russie). Enfin un avis de la Cour Internationale de la Justice en juillet 2010 considère que la déclaration d'indépendance du Kosovo n'était pas contraire au droit international, alors que le rattachement de la Crimée à la Russie est une violation du Traité de Budapest de 1994 par lequel l'Ukraine abandonnait l'arme nucléaire en échange de la garantie par les USA, la Grande Bretagne et la Russie de son intégrité territoriale.

L'Union européenne a été pour les pays d'Europe centrale comme demain pour les Balkans un "substitut d'Empire", un toit commun après la disparition des empires d'antan. Dans son voisinage oriental elle découvre une situation à la fois, post-soviétique et néo-impérial, un entredeux qui, pour les uns, incite à la prudence en Ukraine par une sorte de "finlandisation" et, pour les autres, au contraire à une redéfinition de la politique de voisinage dans le sens d'une possible intégration. Les deux options invoquent des arguments des arguments liant démocratisation et géopolitique: la "finlandisation" permettait un développement démocratique interne en échange d'une neutralité en politique extérieure; les adversaires rappellent que la leçon des crises de 2004 à 2014 
est bien qu'un développement démocratique à l'ombre d'une Russie de plus en plus autocratique ressemble à la quadrature d'un cercle et que seul un encrage européen à terme pourra garantir le développement démocratique en Ukraine et plus généralement dans le voisins oriental de I'UE. Mais les deux arguments convergent pour dire que la réponse doit appartenir aux Ukrainiens.

\section{Conclusions}

Les plaques tectoniques de l'après 1989 sont en train de bouger. La crise ukrainienne a mis en évidence une nouvelle donne dans les relations entre l'UE et la Russie et invite à une reconsidération de la politique de voisinage.

En un mois entre la chute du régime Yanukovitch et l'annexion de la Crimée à la Russie Vladimir Putin a réussi a transformer ce qui était au départ le choix entre deux ensembles commerciaux en un choix géopolitique et "civilisationnel". C'est en ces termes que le président russe a lui même formulé les enjeux et, en bafouant l'intégrité territoriale de l'Ukraine, affirmé la puissance d'une Russie qui se pose en s'opposant à l'Europe. A la fin des années quatre-vingt. Gorbatchev avait formulé le projet d'une "Maison commune européenne" où la Russie aurait sa place. Considérant que ce projet mena "à la plus grande catastrophe géopolitique" du siècle dernier (la fin de l'URSS) Poutin revient en force à une idée ancienne où se mêle la logique impériale et le discours sur la citadelle assiégée. Adam Michnik, l'ancien dissident à Varsovie, en conclut c'est "la fin du quart de siècle le plus faste de l'histoire polonaise depuis quatre siècles"76 La plus faste de l'histoire centre-européenne depuis 1914 sans aucun doute. 
Nous assistons, en effet, à la fin d'une époque, celle de l'après-1989 en Europe. Rétrospectivement on mesure mieux encore le "miracle" que constituait une dissolution pacifique de l'empire soviétique. La guerre de dissolution de la Yougoslavie était là pour nous rappeler que cela n'allait pas de soi. Il y avait, en effet, tous les ingrédients réunis pour les contentieux et conflits liés à la logique de sécession et des "guerres de souveraineté" que résume bien la formule du macédonien V.Gligorov de 1991:"Pourquoi serais-je une minorité dans ton Ełat quand tu peux être une minorité dans le mien". II y avait alors 25 millions de Russes en dehors des frontières de la Russie et donc un risque que la défense des "pieds rouges" puisse s'apparenter à la défense par Belgrade des Serbes dans les républiques nouvellement indépendantes. La différence c'est que Eltsyne n'était pas Milosevic puisque c'est en tant que président élu de la Russie, opposé à Gorbatchev, qu'il donna le dernier coup de boutoir à I'URSS. Poutine a refermé cette la parenthèse et revient par la force à la logique de l'empire.

L'Europe (re)découvre les limites du droit, mais aussi celles de son "soft power". Personne, bien entendu, n'a envie d'un "remake" de la Guerre de Crimée de 1854 et de la "Charge de la brigade légère" glorifiée dans le poème de Tennyson: "Theirs not to make reply, Theirs not to reason why, Theirs but to do and die"... Personne n'ira mourir pour la Crimée, et la prise de Sébastopol par Poutine paraît aux Européens comme un terrible anachronisme: prise d'un territoire, la Crimée, contre la perte d'influence en Ukraine en Europe. L'Ukraine doit inciter I'UE à repenser sa politique de voisinage (accès au marché européen en échange de normes partagées) $)^{77}$. L'Europe n'a pas la géopolitique dans son ADN, elle s'est construite contre la logique de puissance responsable de son autodestruction il y a un siècle. Elle découvre aujourd'hui les limites de la puissance "normative" confrontée à celle d'une Realpolitik postimpériale. II se peut que, confrontée aux nouvelles menaces dans son voisinage Sud et Est, l'islam radical et la résurgence d'un nationalisme russe sous Poutine, une cohésion, une politique de voisinage et de sécurité commune. Si l'Europe veut avoir une influence (géo)politique dans son voisinage, si elle a l'ambition d'être une "Europe espace" elle devra devenir une "Europe puissance". 
Jacques Rupnik (jacques.rupnik@sciencespo.fr) was born in Prague in 1950, educated at the University of Paris and at Harvard, is currently Director of Research at CERI and Professor at Sciences $P o$ in Paris and visiting professor at the College of Europe in Bruges. He holds degrees in history from the University of Paris I-Sorbonne and politics from the Institut d'Etudes Politiques de Paris (IEP), a Master's in Soviet Studies from Harvard University (1974) and a Ph.D. in history from the University of Paris I - Sorbonne (1978). J.Rupnik has been an advisor to the President of the Czech Republic, Vaclav Havel (1990-1992) and continued to work with him after that. He has published a number of books and scholarly articles including The Other Europe (London, 1989, translated to a dozen languages and a companion volume to a six hour documentary film on postwar Eastern Europe for British television). 
? .

x

N

N

$\vec{v}$ 